\title{
RHIC GAMMA TRANSITION JUMP POWER SUPPLY PROTOTYPE TEST*
}

\author{
Jianlin Mi, G. Ganetis, W. Louie, D. Bruno, G. Heppner, J. Sandberg, R. Zapasek, W. Zhang \\ Collider - Accelerator Department, Brookhaven National Laboratory Upton, NY 11973-5000, USA
}

\begin{abstract}
This paper describes the principle and test result of the prototype RHIC Gamma Transition Jump Power Supply. The jump power supply principle is introduced and illustrated with diagram in this paper. The prototype is built with Insulated Gate Bipolar Transistors (IGBT) as current direction switch components. Optically coupled IGBT drives are used for the jump control switch. The jump time is synchronized from 40 to 60 millisecond to meet the RHIC beam transition-crossing requirement. The short jump time is needed to avoid particle loss and to preserve the initial bunch area during the transition, thus successfully transferring the ion beams from the acceleration RF system to storage system. Total twenty four jump power supplies will be used, they synchronously switch the direction of the magnets current while the beam is being accelerated through the transition to reach the top storage energy. Each power supply will energize a group of super conducting magnets, which consists of four magnets that connected in series. At the end, test results are listed, accompanied with the dummy load current waveform and prototype picture.
\end{abstract}

\section{INTRODUCTION}

Protons and ions are accelerated and stored in the Relativistic Heavy Ion Collider (RHIC). With the exception of protons, all ions injected into RHIC form Alternating Gradient Synchrotron (AGS) are below the transition energy. And consequently, it has to be accelerated through transition to reach the top energy for storage. The acceleration is provided by the RF system. In order to successfully transfer the ion beams from the injection energy to the storage energy, it is of primary importance to avoid particle loss and to preserve the initial bunch area during the transition crossing.

It has been shown that the transition crossing can be achieved with no particle loss and negligible bunch-area growth, when a gamma transition jump is employed in a time period of $40 \mathrm{~ms}$ to $60 \mathrm{~ms}$.

There are twelve sextants at the RHIC, six sextants in blue ring and another six sextants in yellow ring. There are eight gamma transition jumping quadruple magnets at each sextant.

At each sextant, two power supplies energize eight magnets. First two magnets and last two magnets connected in series are powered by a jump power supply and another left four magnets connected in series are energized by another jump power supply. The layout of the magnets and power supplies at one sextant are shown in figure 1. Both yellow and blue super conducting magnets rings employs total 94 quadruples magnets.

\footnotetext{
*Work performed under the auspices of the U.S. Department of Energy
}

There are total twelve power supplies for each super conducting ring $\bullet T$ jumping system. There are total 24 power supplies driving the twelve sextant magnets.

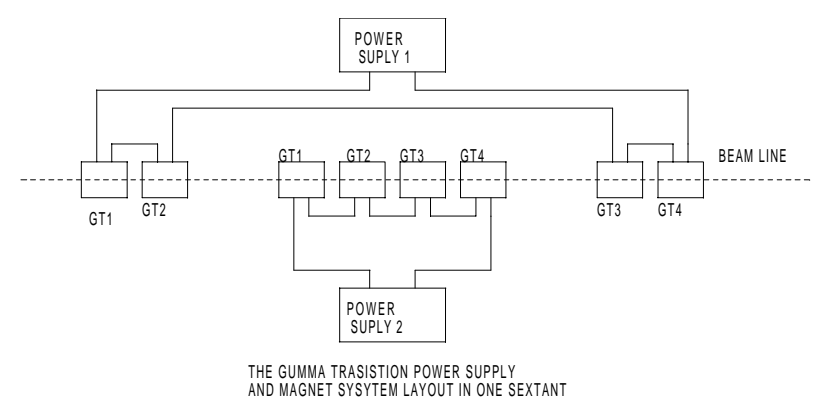

Figure 1: $\gamma$ T P.S. and Magnet system Layout Diagram

\section{$2 \gamma$ T JUMP P.S. REQUIREMENT AND SPECIFICATION}

$\gamma \mathrm{T}$ Jump Power Supply is a current negative feedback power supply used for energize four quadruple magnets. The peak output current is of $\pm 40 \mathrm{~A}$. The operating current amplitude demanded is depending on the magnetic field, which tracks the ion beam energy in the RHIC storage ring. The power supply output current amplitude should follow the tracks of the ion beam energy from injection energy to storage energy. The power supply should have current direction jump function, to avoid beam loss, from positive direction to negative direction or from negative to positive, it depend on the quadruple magnet location. The current direction jumping should complete during a time of 40 millisecond to 60 millisecond. The required magnet current waveform is shown in figure 2 . The power supply main specification data is following:
Total power supply

Maximum out put voltage

Out put Current

Current jump time

Out put current stability

Load inductance around

Load resistance

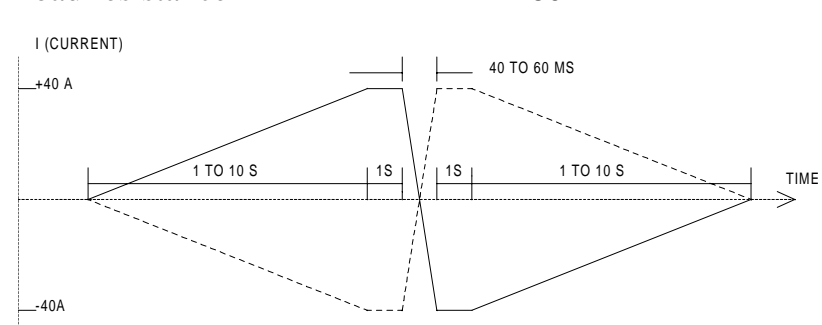

POWER SUPPLY OUTPUT CURRENT WAVEFORM
24 (12 P.S. each ring)

$20 \mathrm{~V}$

$\pm 40 \mathrm{~A}$

40 to $60 \mathrm{~ms}$

$0.1 \%$

$120 \mathrm{mH}$

$260 \mathrm{~m} \Omega$
Figure 2: GT P.S. Current Waveform Demand 


\section{THE PRINCIPLE OF THE $\gamma$ T JUMP POWER SUPPLY}

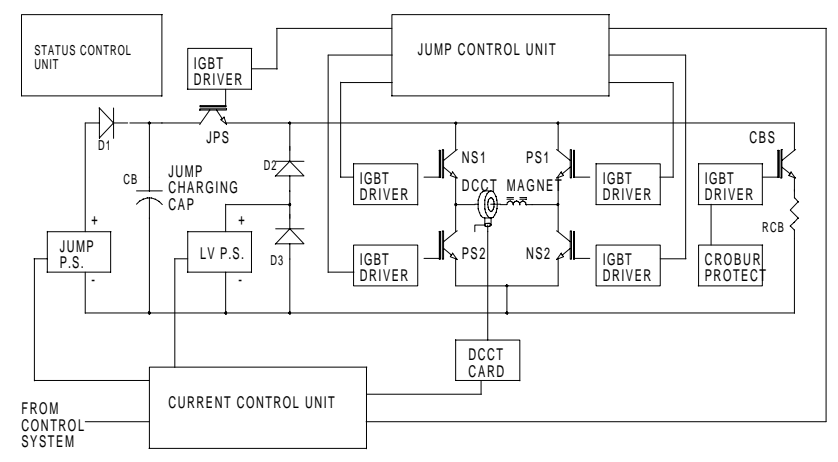

Figure 3. GT Power Supply Diagram

Figure 3 shows the gamma transition power supply diagram. Four IGBT, (NS1, NS2, PS1 and PS2) consist of a current direction switch bridge to output a polarity changeable current to the magnet load. The IGBT pair PS1 and PS2 conducts positive direction current. The negative direction current is delivered by another IGBT pair (NS1 and NS2). A lower voltage power supply (LV P.S.) is used as the main power supply, which can deliver enough output current to current direction switch bridge through a diode D2. The current control unit controls the current output. The current amplitude is depended on the current reference from the control system. The current waveform should match the beam acceleration ramping requirement. The power supply load is four magnets corrected in series with a high inductance $(120 \mathrm{mH})$. The load high inductance limits the current polarity jump time. A high voltage power supply is needed for increase the current $\mathrm{di} / \mathrm{dt}$ rate while the magnet current switch from one direction to another direction. A $600 \mathrm{~V}$ dc power supply is employed to charge a capacitor bank (CB), which is consisted of four dielectric capacitors. While the current switch bridge changing the current polarity direction, a moderate long pulse is sent to the IGBT JPS driver to turn on the JPS IGBT. A high enough voltage is added crossing the inductance load to increase the load current $\mathrm{di} / \mathrm{dt}$ rate and foreshorten the current changing time form one polarity direction to another. When the current, which is detected by DCCT, reaches the same absolute amplitude, the jump controller unit turns off the IGBT JPS. And then the load current is delivered from the lower voltage power supply through the IGBT bridge. The current polarity changing is completed. During the current jumping time the low voltage power supply (LV P.S.) keeps the same out put voltage.

\section{PROTOTYPE MAIN PARTS}

There are several main parts are chosen for the prototype.

\subsection{Lower Voltage and Jump Power Supply}

A XANTREX Model 20-60 lower voltage power supply is used as the main power supply, which is a low noise, precisely regulated, variable DC power supply.
This is a programming power supply. It can provide a $20 \mathrm{~V}, 60 \mathrm{~A}$ DC current in voltage or current regulated model. A small DC to DC $1 \mathrm{KV}$ power supply, model 1C24-P60 from ULTRAVOLT INC, is used as the jump power supply to charging the jump capacitor bank, This high voltage power supply operating voltage is setting at an output voltage less than $600 \mathrm{v}$.

\subsection{IGBT and Driver}

Six IGBT NS1, NS2, PS1 PS2, JPS1 and CBS are manufactures by the POWEREX. Three PM150DSA 120 IGBT modules are chosen in this power supply. Each module has two IGBT connected in series. This is an intellimode intelligent high output power IGBT module. Each IGBT can deliver 150 Amperes, with a 1200 voltage collector emitter voltage. This is an isolated base module designed for power switching applications to $20 \mathrm{kHZ}$. Built in control circuits provide optimum gate drive and protection for the IGBT and free wheel diode power device.

Three IGBT driver boards (APS-1036) from APS (Applied Power System Co.) are chosen to excite the IGBT. It is an optically coupled isolation IGBT driver. There are two IGBT drivers on one board. Each driver board can excite one IGBT module.

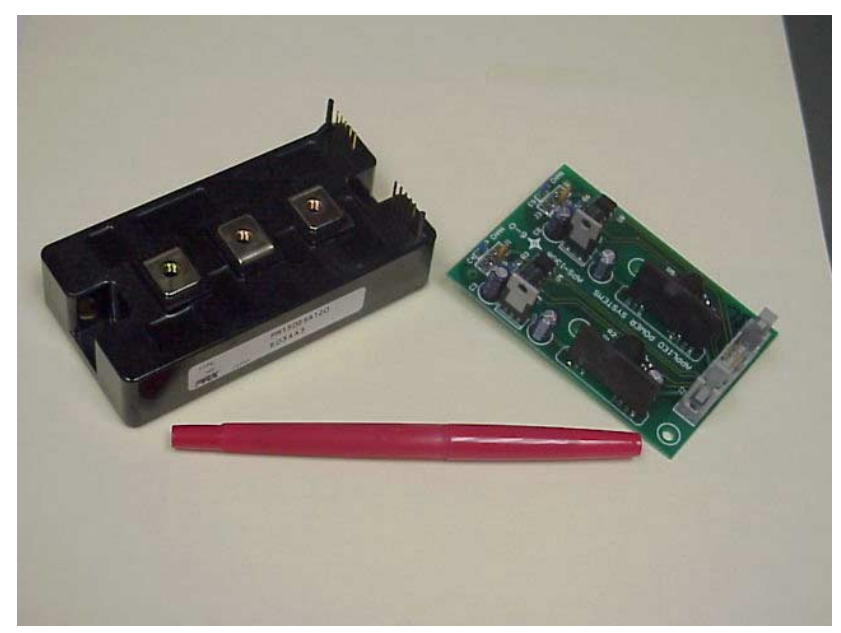

Figure 4: IGBT PM150DSA120 and IGBT Driver.

\subsection{Control Cards and Buffer Board.}

Control system consists of seven control cards divided into three functions. The three functions are power supply status control; current regulator control and current jump control.

The status control unit includes Control Card and Digital Isolation Card. This unit manipulates the power supply ON/OFF and interlock protection. The protection involves fan air flowing stop interlock; IGBT heat sink over temperature; IGBT over current; capacitor over voltage protection and etc.

Current control unit includes DCCT card, analog signal buffer card and current regulator card.

Jump control card control the current polarity switch bridge and jump switch. And a magnet load over voltage protection or call the super conductor quench protection is 
necessary. If the voltage escalates to high than the setting value, the quench protection will act and the jump control card sends a fire pulse to the CBS dumping the magnet energy to the dumping resistor RCB

An Isolation Buffer Amplifier Board is installed in the power supply chassis for signal processing and communication between the power supply and the control units.

\section{PROTOTYPE TEST RESULTS}

The gamma transition jump power supply prototype was assembled and tested. Figure 5 shows the power supply prototype chassis. An inductor dummy load was connected to the power supply prototype. The power supply can follow the input reference voltage very good and the current stability fits the specification. The test concentrates on the current switch and polarity jumping. The power supply maximum out put current can reach $\pm 60 \mathrm{~A}$ and the current jumping time is $40 \mathrm{mS}$. The current jump overshot is less than $1 \%$. One of the dummy load current jumping waveforms is shown at figure 6 .

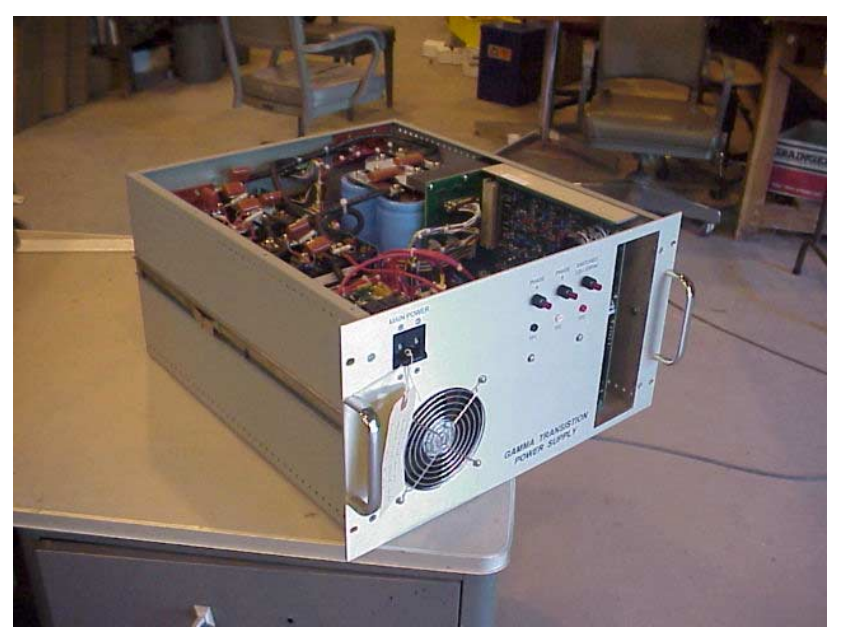

Figure 5: Picture of Power Supply Prototype.

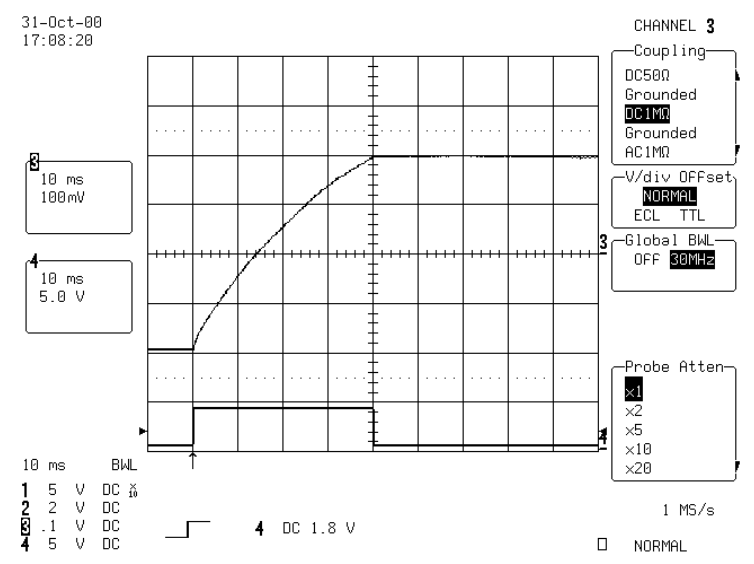

Figure 6: Damping Load Jump Current Waveform (10A/div, 10ms/div)

\section{CONCLUSIONS}

The prototype power supply test fits the design requirement. The current jump time can be controlled from $40 \mathrm{~ms}$ to $60 \mathrm{~ms}$. Total 24 gamma transition power supplies has been manufactured from the vender. The power supplies are being installed into RHIC ring. Each power supply should be tested before it is installed into the ring. The power supply system commission will be proceeding soon.

\section{ACKNOWLEDGEMENTS}

The authors would like to express their thanks to R. Lambiase for his helping on the circuit simulation; they would like to thank G. Heppner for his valuable contribution on prototype assembling and testing; $\mathrm{M}$. DeLavVergne for his ingenious chassis parts layout arrangements, J. Wilke for the control cards measurement and other friends helps at Collider - Accelerator Department.

\section{REFERENCES}

[1] Collider-Accelerator Department, BNL, "RHIC Design Manual",

[2] J. Kewisch, "Gamma Transition Jump with quadrupole"

[3] W. Louie, "RHIC Gamma Transition Power System" 